OPEN ACCESS

Edited by:

Ye Zeng,

Sichuan University, China

Reviewed by:

D. Neil Granger,

Louisiana State University Health

Shreveport, United States

Eric Schmidt

University of Colorado, Denver, United States

*Correspondence: Sarah Y. Yuan syuan@usf.edu

Specialty section:

This article was submitted to

Signaling,

a section of the journal

Frontiers in Cell and Developmental

Biology

Received: 17 May 2021

Accepted: 22 June 2021

Published: 14 July 2021

Citation:

Villalba N, Baby S and Yuan SY (2021) The Endothelial Glycocalyx as

a Double-Edged Sword

in Microvascular Homeostasis

and Pathogenesis.

Front. Cell Dev. Biol. 9:711003.

doi: 10.3389/fcell.2021.711003

\section{The Endothelial Glycocalyx as a Double-Edged Sword in Microvascular Homeostasis and Pathogenesis}

\author{
Nuria Villalba ${ }^{1}$, Sheon Baby ${ }^{1}$ and Sarah Y. Yuan ${ }^{1,2 *}$ \\ 1 Department of Molecular Pharmacology and Physiology, Morsani College of Medicine, University of South Florida, Tampa, \\ FL, United States, ${ }^{2}$ Department of Surgery, Morsani College of Medicine, University of South Florida, Tampa, FL, \\ United States
}

Expressed on the endothelial cell (EC) surface of blood vessels, the glycocalyx (GCX), a mixture of carbohydrates attached to proteins, regulates the access of cells and molecules in the blood to the endothelium. Besides protecting endothelial barrier integrity, the dynamic microstructure of the GCX confers remarkable functions including mechanotransduction and control of vascular tone. Recently, a novel perspective has emerged supporting the pleiotropic roles of the endothelial GCX (eGCX) in cardiovascular health and disease. Because eGCX degradation occurs in certain pathological states, the circulating levels of eGCX degradation products have been recognized to have diagnostic or prognostic values. Beyond their biomarker roles, certain eGCX fragments serve as pathogenic factors in disease progression. Pharmacological interventions that attenuate eGCX degradation or restore its integrity have been sought. This review provides our current understanding of eGCX structure and function across the microvasculature in different organs. We also discuss disease or injury states, such as infection, sepsis and trauma, where eGCX dysfunction contributes to severe inflammatory vasculopathy.

Keywords: inflammation, microvascular homeostasis, permeability, endothelium, glycocalyx

\section{INTRODUCTION}

The vascular endothelial surface is coated by the GCX matrix that confers important functions in circulatory homeostasis (Weinbaum et al., 2007). The endothelial GCX (eGCX), first visualized in the late 1960s after the invention of transmission electron microscope (Luft, 1966), is mainly formed by proteoglycans and glycoproteins, core proteins anchored to the EC membrane that serve as a foundation for the rest of the glycocalyx constituents. Proteoglycans, principally syndecans and glypicans, are decorated by glycosaminoglycan (GAG) chains such as heparan sulfate and chondroitin sulfate (Li et al., 2012). GAGs are characterized by long linear polysaccharides of repeating disaccharide units with a hexosamine and either an uronic acid or a galactose

Abbreviations: BBB, blood-brain barrier; DAMP, danger-associated molecular pattern; EC, endothelial cell; eNOS, endothelial nitric oxide synthase; eSOD, endothelial superoxide dismutase; GAG, glycosaminoglycan; eGCX, endothelial glycocalyx; LPS, lipopolysaccharide; NO, nitric oxide; ROS, reactive oxygen species; SAE, sepsis-associated encephalopathy; TRPs, transient receptor potential channels. 
(Esko et al., 2009). The amount of GAG chains, length and molecular modifications by sulfation and/or (de)acetylation provide the eGCX an extensive source of structural rearrangements. Notably, heparan sulfate proteoglycans are the most prominent members expressed on the surface of the endothelial cells, accounting for $50-90 \%$ of the total endothelial proteoglycans (Ihrcke et al., 1993). The majority of the interactions between syndecans and extracellular matrix molecules, growth factors and cell adhesion molecules seem to be mediated by their heparan sulfate chains through electrostatic interaction (Bernfield et al., 1992; Stringer and Gallagher, 1997). Unlike other eGCX constituents, hyaluronic acid is a linear, non-sulfated GAG that interacts with the cell surface receptor CD44, a glycoprotein (Aruffo et al., 1990). The glycoproteins are highly branched short carbohydrate chains (2-15 sugar residues) capped with sialic acid or a fucose, which mainly function as either endothelial adhesion molecules or components of the coagulation system (e.g., selectins, immunoglobulins, and integrins) (Figure 1). Further detailed structure and specific components of the eGCX are reviewed elsewhere (Pries and Kuebler, 2006; Tarbell and Pahakis, 2006; Reitsma et al., 2007; Weinbaum et al., 2007; Esko et al., 2009). It is worth noting that the eGCX composition is subject to a highly dynamic regulation and constant replacement or re-arrangement of molecules, ranging from enzymatic degradation ("shedding") to de novo biosynthesis of new molecules and to recruitment of circulating molecules from the blood.

In the following sections, we will focus our discussion on the eGCX as an active component of the EC barrier, its functions, and structural variations within the vascular tree and across organs. Furthermore, we will also summarize the new findings from eGCX research with respect to how eGCX degradation leads to certain vascular pathologies.

\section{The eGCX: An Active Layer Without a Passive Role}

The eGCX matrix is an integral component of the vascular wall. Apart from being a physical barrier, the eGCX also plays an effective role in modulating vascular homeostasis. Historically, the eGCX was considered to function as an additional physical barrier between the vessel lumen and the EC membrane (Curry and Adamson, 2012); however, solid experimental evidence has shown an important physiological role for the eGCX in performing a variety of microvascular functions such as regulating vascular permeability, mechanotransduction and leukocyte transmigration (Ihrcke et al., 1993; Davies, 1995; Baldwin and Thurston, 2001; Constantinescu et al., 2003; Curry, 2005; Tarbell and Ebong, 2008; Lopez-Quintero et al., 2009; Lennon and Singleton, 2011; Curry and Adamson, 2012).

The eGCX is one of the major determinants in maintaining endothelial barrier function by acting as an additional molecular filter for the endothelium. The eGCX modulates vascular permeability and hydraulic conductivity by limiting the flux of water and macromolecules (Curry and Michel, 1980; Adamson, 1990; Curry, 2005; Lennon and Singleton, 2011; Curry and
Adamson, 2012). It also acts as a vascular barrier through modulation of molecular binding to the EC surface due to the high density of anionic charges on its GAGs side chains. The net negative charge of the eGCX carried by sulfate residues along the GAG chains favors the docking (adsorption) of positively charged molecules (Schnitzer, 1988; Lieleg et al., 2009). Thus, the eGCX regulates vascular permeability by restricting circulating molecules from strongly attaching to the endothelium based on their net charge. Importantly, the molecular size (70$\mathrm{kDa}$ cutoff) is also relevant in determining the penetration of molecules into the eGCX layer, as much as chemical binding (Henry and Duling, 1999; Vink and Duling, 2000; Curry and Adamson, 2012).

Previous studies using perfusion models or intravital microscopy techniques found that eGCX damage by heparinase causes microvascular leakage (Rehm et al., 2004; Jacob et al., 2006). Similar results were found using genetic knock down of a specific eGCX component (Voyvodic et al., 2014). In this regard, increased hydraulic conductivity $(L p)$ of microvessels after removal of the eGCX or plasma proteins has also been shown (Huxley and Curry, 1985; Adamson and Clough, 1992; Weinbaum et al., 2007).

The eGCX plays a pivotal role in mechanotransduction together with other sensors in the endothelium, including Gprotein-coupled receptors (Zou et al., 2004; Mederos y Schnitzler et al., 2008), Piezo and transient receptor potential (TRP) channels (Martinac, 2004; Coste et al., 2010; Dragovich et al., 2016), caveolar structures (Rizzo et al., 1998), and integrins and focal adhesions (Ringer et al., 2017). Blood flow exerts mechanical tangential forces to the endothelial surface such as shear stress, which is sensed by the eGCX and triggers the production of nitric oxide (NO), an important modulator of vascular tone (Davies, 1995; Dimmeler et al., 1999; Tarbell and Ebong, 2008; Fu and Tarbell, 2013; Zeng et al., 2018). The ability of the eGCX to reorganize the actin cytoskeleton under shear forces has been demonstrated in studies using EC monolayers as well as in vivo approaches. The eGCX core protein syndecan-1 interacts with cytoskeletal proteins through a highly conserved tyrosine residue in the syndecan family (Carey et al., 1996). Also, syndecan4 acts synergistically with integrins to assemble and rearrange actin stress fibers to orchestrate cell adhesion and focal contact formation (Echtermeyer et al., 1999; Bass et al., 2007; Multhaupt et al., 2009). Interestingly, while syndecans are the main effector in cell adhesion or shape changes via their interaction with the cytoskeleton, glypicans mediate flow-induced endothelial NO synthase (eNOS) activation, based on their location at the endothelial membrane microdomains where caveolae reside (Ebong et al., 2014; Zeng and Liu, 2016; Bartosch et al., 2017). Prior studies with cultured ECs have shown that breakdown of heparan sulfate alters shear stress and impairs NO production (Florian et al., 2003); similar responses were also observed in vivo on canine femoral and rabbit mesenteric arteries, where infusion of hyaluronidase (to degrade hyaluronic acid GAGs) or neuraminidase (to remove sialic acid residues), respectively, reduced flow-dependent vasodilation, which is mediated by NO release (as in the majority of vascular beds) (Pohl et al., 1991; Mochizuki et al., 2003). 


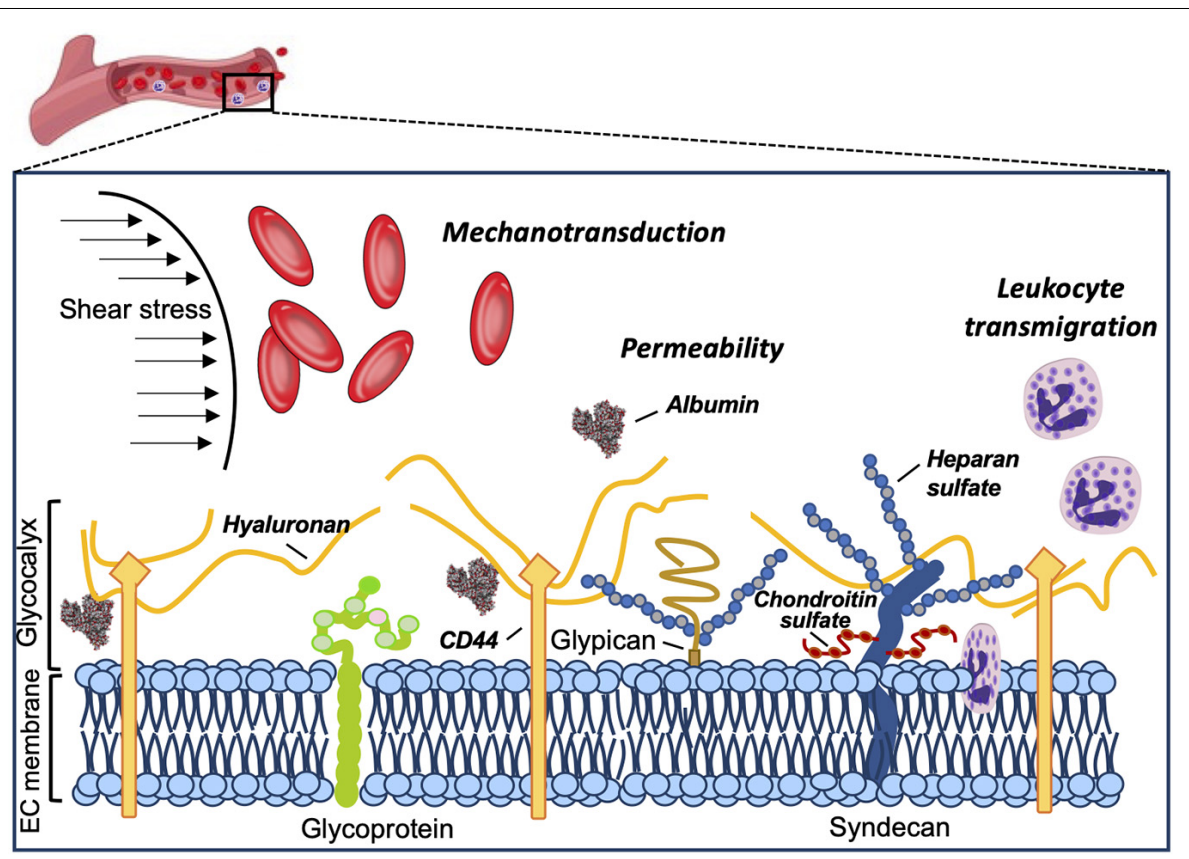

FIGURE 1 | Structure and functions of the eGCX. Schematic representation of the main components and functions of the endothelial glycocalyx. The eGCX is composed of proteoglycans, with long glycosaminoglycan side-chains (GAG-chain) and glycoproteins, with short branched carbohydrate side-chains. The eGCX modulates coagulation, inflammation and mechanotransduction processes.

Additionally, the eGCX also controls the interaction between the endothelium and circulating cells by preventing the latter from approaching the endothelium under basal conditions. Upon inflammatory stimulation, the glycans are shed from the EC surface allowing slow rolling and adhesion of leukocytes (Constantinescu et al., 2003; Lipowsky et al., 2011). Similarly, breakdown of the eGCX increases platelet-vessel wall interactions, further demonstrating an anti-coagulant effect by the eGCX layer (Vink et al., 2000).

\section{The Endothelium Is Heterogenous, So Is the eGCX}

The morphology of the microvascular endothelium and associated gene expression vary across different vascular beds in different tissues, therefore showing a remarkable heterogeneity (Aird, 2007; Jambusaria et al., 2020). Likewise, different GAG chain arrangements and eGCX compositions result in great biochemical or structural variations, further contributing to the eGCX heterogeneity. With reference to the thickness and microstructure of the eGCX, it is now well established that both vary across different species, vascular beds, organs and shear stress rates.

The estimation of the eGCX thickness extends from 0.2 to $0.5 \mu \mathrm{m}$ in capillaries (van den Berg et al., 2003) and venules (Yoon et al., 2017), to 2-3 $\mu \mathrm{m}$ in small arteries (van Haaren et al., 2003; Yen et al., 2015), and $4.5 \mu \mathrm{m}$ in conductance arteries (Megens et al., 2007). These studies used different methods of eGCX visualization and measurements, including alcian blue staining for transmission electron microscopy, dye-exclusion of different sized tracers, and fluorescently labeled lectins for microscopic imaging (Roth, 1983; Vink and Duling, 2000; van den Berg et al., 2003). Still, there is a large discrepancy when it comes to reporting eGCX thickness, making experimental observations particularly difficult to be reconciled. The reason for this variability, which might not be entirely attributed to differences in the microstructure and composition of the eGCX, might rather be due to a poor preservation of such a fragile structure during fixation and tissue handling (de Mesy Bentley, 2011; Ebong et al., 2011). Comparatively, direct in vivo measurements using bright-field microscopy also embody challenges. The close optical refractive index of the eGCX to the surrounding blood makes it very difficult to visualize the eGCX limits, also contributing to bias in the results. In vitro, ECs in culture exhibit slightly different eGCX in comparison to the complex structure found in in vivo vessels (Potter and Damiano, 2008; Potter et al., 2009). Recently, super resolution fluorescence microscopy (STORM) has been applied to identify the spatio-chemical organization of the eGCX in vitro (Fan et al., 2019). Also, glycomic analysis by liquid chromatography coupled to mass spectrometry has emerged as a novel method providing a more detailed and comprehensive characterization of eGCX in cells and tissues (Li et al., 2019, 2020; Riley et al., 2020).

A close view of the eGCX using both scanning and transmission electron microscopy has revealed different eGCX thickness among continuous, fenestrated and sinusoidal capillaries in the heart, kidney, and liver, respectively (Okada et al., 2017). The eGCX layer in both continuous and fenestrated capillaries is thicker than in the sinusoids. In the heart, the eGCX covers the entire luminal endothelial surface. In the kidney, the eGCX appears to occlude the endothelial pores of the fenestrated capillaries. In the hepatic sinusoids, however, the eGCX covers 


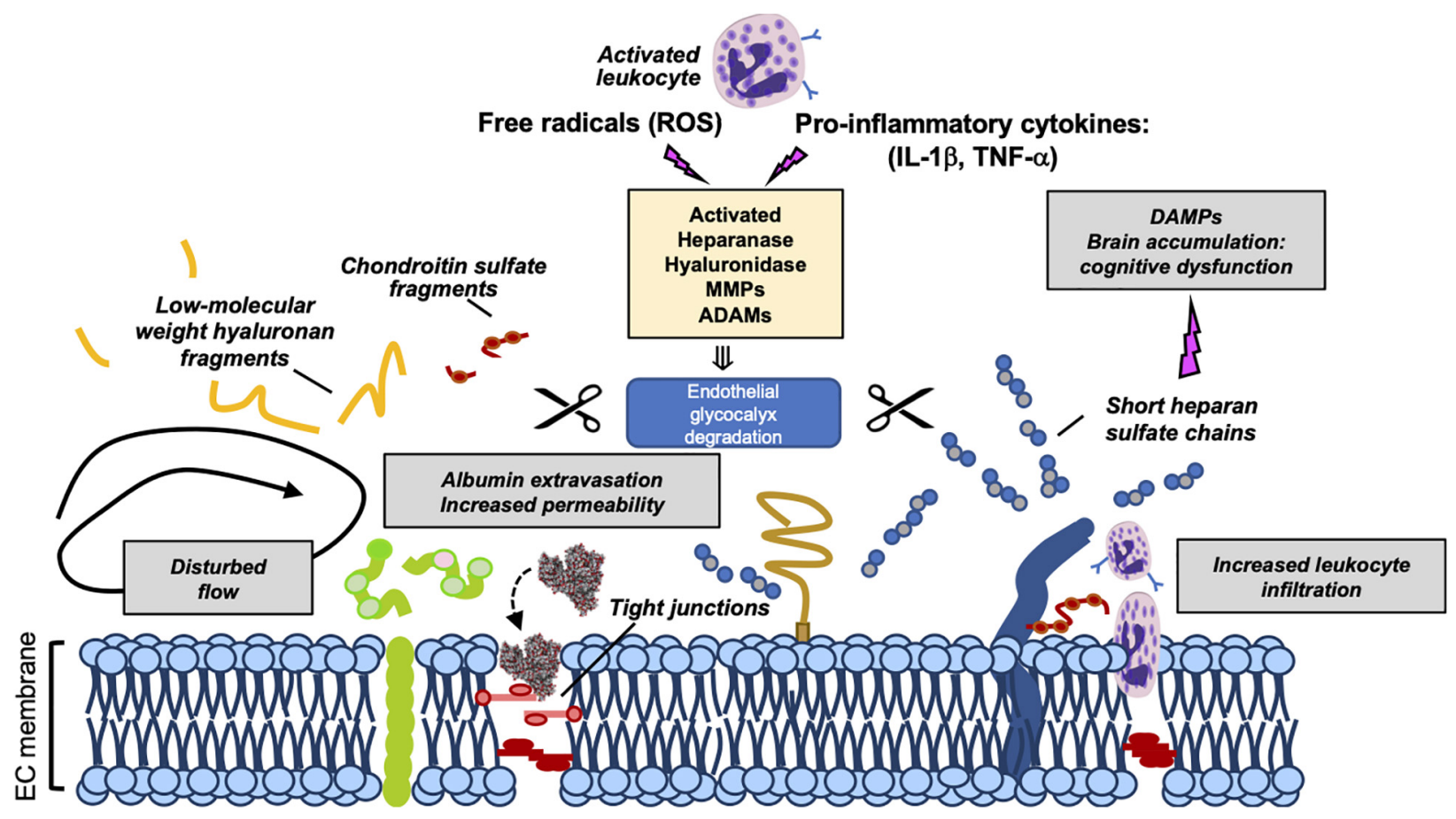

FIGURE 2 | Mechanisms of eGCX degradation and pathogenic consequences of released GCX fragments. Representation of enzymatic degradation of GCX components. The structure of the eGC is the result of a balance between the enzymatic degradation and de novo biosynthesis of new molecules and adsorption of circulating components from blood. Several enzymes mediate this degradation. Heparinase, hyaluronidase, MMPs and ADAMs are activated by pro-inflammatory cytokines and ROS promoting the damage and shedding of one or more of its components. This degradation releases eGCX components (such as short heparan sulfate chains, low-molecular weight hyaluronan fragments, and chondroitin sulfate fragments) into the circulation. As a result of its degradation, the eGC becomes thinner allowing the extravasation of albumin, leukocyte adhesion and dysregulated vasodilation. Once in circulation, eGCX components such as heparan sulfate fragments can act as DAMPs leading to cognitive impairment (Hippensteel et al., 2019a). Gray box areas summarize major pathophysiologic features of eGCX degradation. DAMPs, danger-associated molecular patterns; MMP, metalloproteinase; IL-1 $\beta$, interleukin- $1 \beta$; TNF- $\alpha$, tumor necrosis factor- $\alpha$. Scissors symbol means "degradation".

both the luminal side and opposite side facing the perisinusoidal space (Okada et al., 2017).

In organs like the brain and heart, where the capillary endothelium is categorized as continuous (non-fenestrated), the endothelial eGCX appears to be denser compared to that in the lung, whose capillaries are also covered by continuous endothelium (Ando et al., 2018). These differences might be explained by the mechanotransduction properties of the eGCX in sensing fluid shear stress, which alters GAGs synthesis (Arisaka et al., 1995; Gouverneur et al., 2006; Zeng and Tarbell, 2014). Since the pulmonary circulation is a low fluid shear stress system (because of its low resistance), a lower rate of GAGs synthesis renders a thinner eGCX on the pulmonary capillaries compared to other organs like the heart or the kidney. However, experimental evidence shows discrepancies in eGCX depth between pulmonary eGC ( $>1.5$ micrometers) exceeding that of systemic vessels such as the eGCX in cremaster muscle capillaries (Schmidt et al., 2012; Han et al., 2016). The same principle can be applied to the macro vs. microvascular network, where arteries receiving higher shear stress exhibit greater eGCX depths compared to venules and capillaries with lower shear stress (Lipowsky et al., 1978, 1980; van den Berg et al., 2003). In light of recent discoveries, differences in capillary EC structure and shear stress might not be sufficient to explain eGCX heterogeneity. Gene expression profiling and single-cell RNA-sequencing might yield a more comprehensive picture of the distinct EC subsets and associated eGCX structures (Jambusaria et al., 2020; Gao and Galis, 2021).

\section{Severe Inflammation as a Cause of eGCX Dysfunction}

Recently, the eGCX integrity has emerged as an important determinant of cardiovascular health and disease. Given the fundamental role of the eGCX in maintaining vascular homeostasis, one would predict that when components of the eGCX are lost or degraded, the endothelial function could be impaired, which has indeed been demonstrated. eGCX degradation is triggered by inflammatory mechanisms through the activation of specific enzymes such as metalloproteinases, heparanase, and hyaluronidase. These enzymes are activated by reactive oxygen species (ROS) and pro-inflammatory cytokines such as tumor necrosis factor alpha (TNF- $\alpha)$ and interleukin1 beta (IL-1 $\beta$ ) (Figure 2) (Chappell et al., 2008; Schmidt et al., 2012; Lipowsky and Lescanic, 2013; Manon-Jensen et al., 2013; Becker et al., 2015). 
The lack of an intact eGCX has been observed in several pathological conditions, the best characterized being sepsis. In the broad scheme of sepsis, systemic inflammatory injury of the eGCX leads to capillary leak, adverse immune response, and impaired vasodilation. Following septic challenge, enzymes such as ADAM15 (a disintegrin and metalloproteinase 15) and heparanase can shed glycoproteins (CD44) and heparan sulfate, respectively, leading to eGCX disruption (Schmidt et al., 2012; Yang et al., 2018). As a result of eGCX damage, the eGCX layer becomes thinner and more sparse while its degradation products are released into the bloodstream, a phenomenon that has been observed in animal models of sepsis as well as in human patients with sepsis, trauma or shock (Nelson et al., 2008; HaywoodWatson et al., 2011; Sallisalmi et al., 2012; Luker et al., 2018; Uchimido et al., 2019).

Similar to sepsis, sterile inflammation following trauma or tissue injury also causes shedding of proteoglycans, hyaluronan and heparan sulfate chains. The eGCX fragments function as Danger-Associated Molecules Patterns (DAMPs) that activate toll-like receptor or/and RAGE receptor-dependent pathways (Johnson et al., 2002) RAGE (Xu et al., 2011, 2013). High levels of circulating eGCX elements, which propagate sterile inflammation and drive trauma induced coagulopathy (TIC), are highly correlated with the severity of injury and clinical outcomes (Johansson et al., 2011a,b).

Oxidative stress also plays an important role in eGCX degradation during inflammation. The eGCX along with vascular ECs are vulnerable to circulating ROS produced during oxidative stress. In vitro exposure of ROS (superoxide and hydroxyl radicals) to the eGCX promotes fragmentation of GAGs and loss of some of its components. Previous studies have demonstrated that hyaluronan and chondroitin sulfate are the most susceptible to depolymerization and chemical modifications by ROS (Halliwell, 1978; Greenwald and Moy, 1980; Bartold et al., 1984; Moseley et al., 1995, 1997; Lipowsky and Lescanic, 2013; Singh et al., 2013). Intact eGCX has the capability to quench free radicals by having binding sites for anti-oxidant enzymes like xanthine oxidoreductase (Adachi et al., 1993) and endothelial superoxide dismutase (eSOD) (Becker et al., 1994).

Viral infections, such as those caused by dengue, hanta and the novel severe acute respiratory syndrome (SARS)-CoV2 (COVID-19), are also accompanied by eGCX disruption. In the case of the dengue virus, in particular, the secreted dengue virus (DENV) non-structural protein 1 (NS1) disrupts the eGCX on human pulmonary capillaries by increasing the expression of sialidases, heparanase and metalloproteinases. All these events cause systemic microvascular leakage leading to hypovolemic shock and potentially fatal complications in severe dengue infections (Luplertlop and Misse, 2008; Puerta-Guardo et al., 2016; Glasner et al., 2017; Suwarto et al., 2017; Tang et al., 2017; Chen et al., 2018; Wang et al., 2019). Hantavirus infection is also associated with endothelial dysfunction and elevated circulating levels of syndecan-1, allowing a clinical association of disease severity with eGCX damage (Marsac et al., 2011; Connolly-Andersen et al., 2014). In contrast, other viruses do not seem to cause eGCX shedding, but they exploit eGCX components on the host cell surface as a binding site to infect target cells. For example, Influenza A uses sialic acid as a receptor (Weis et al., 1988; Matrosovich et al., 1993; Suzuki, 2003; Russell et al., 2008) while HIV lentivirus (Saphire et al., 2001; Bobardt et al., 2003; Gallay, 2004) and SARS-CoV-2 (Clausen et al., 2020) interact with heparan sulfate. Also, several recent studies have emphasized the implications of eGCX damage and endothelial dysfunction in the pathogenesis of COVID-19 (Jung et al., 2020; Kaur et al., 2020; Libby and Luscher, 2020; Teuwen et al., 2020; Yamaoka-Tojo, 2020).

Previous research on fluid resuscitation for critical illness management has shown mixed results, some show attenuating eGCX degradation while others show inducing eGCX disruption (Hippensteel et al., 2019b). However, there is consensus that colloids (e.g., albumin), or fresh frozen plasma, reduce eGCX damage following sepsis, hemorrhagic shock and traumatic brain injury (Zehtabchi and Nishijima, 2009; Haywood-Watson et al., 2011; Kozar et al., 2011; Peng et al., 2013; Mica et al., 2016; Nikolian et al., 2018).

\section{Endothelial GCX in Blood-Brain Barrier (BBB) Injury}

The diagnostic utility of eGCX degradation products as a biomarker of disease is supported by the correlation between circulating eGCX fragments and clinical outcomes [reviewed by Uchimido et al. (2019)]. Compared to the cardiac and pulmonary capillaries, cerebral capillaries have a thicker eGCX layer which is better preserved following lipopolysaccharide (LPS) administration (Ando et al., 2018). Additionally, the eGCX joins astrocyte endfeet and basement membrane in reinforcing $\mathrm{BBB}$ properties as a part of a newly defined "tripartite" BBB layered structure (Kutuzov et al., 2018). During sepsis, heparan sulfate fragments released from the injured eGCX can circulate in the bloodstream for days and penetrate into the hippocampal area, interfering with long-term potentiation (LTP) and contributing to sepsisassociated encephalopathy (SAE), a common neurological complication of sepsis in the absence of direct brain infection (Hippensteel et al., 2019a). Circulating eGCX fragments predicted cognitive impairment in septic patients, however, whether they have potential diagnostic utility as biomarkers to predict cognitive dysfunction in sepsis survivors, still remains to be confirmed.

\section{CONCLUSION}

The eGCX, a complex and fragile structure that protects endothelial barrier integrity, plays a crucial role in maintaining microcirculatory homeostasis and blood-tissue exchange. Disruption of eGCX is a consequence as well as cause of microvascular injury, as eGCX degradation products act as pathogenic factors capable of inducing endothelial hyperpermeability and microvascular leakage during inflammation. Further studies are required to understand eGCX structure and function in order to maximize its protective 
contribution to endothelial cell stability while minimizing its pathological role in vascular disease and injury.

\section{AUTHOR CONTRIBUTIONS}

$\mathrm{NV}$ performed literature search, drafted the manuscript, and prepared the figures. SB and SY participated in manuscript editing. SY initiated, directed, and sponsored the work

\section{REFERENCES}

Adachi, T., Fukushima, T., Usami, Y., and Hirano, K. (1993). Binding of human xanthine oxidase to sulphated glycosaminoglycans on the endothelial-cell surface. Biochem. J. 289, 523-527. doi: 10.1042/bj2890523

Adamson, R. H. (1990). Permeability of frog mesenteric capillaries after partial pronase digestion of the endothelial glycocalyx. J. Physiol. 428, 1-13. doi: 10.1113/jphysiol.1990.sp018197

Adamson, R. H., and Clough, G. (1992). Plasma proteins modify the endothelial cell glycocalyx of frog mesenteric microvessels. J. Physiol. 445, 473-486. doi: 10.1113/jphysiol.1992.sp018934

Aird, W. C. (2007). Phenotypic heterogeneity of the endothelium: i. structure, function, and mechanisms. Circ. Res. 100, 158-173. doi: 10.1161/01.RES. $0000255691.76142 .4 \mathrm{a}$

Ando, Y., Okada, H., Takemura, G., Suzuki, K., Takada, C., Tomita, H., et al. (2018). Brain-specific ultrastructure of capillary endothelial Glycocalyx and its possible contribution for blood brain barrier. Sci. Rep. 8:17523. doi: 10.1038/s41598018-35976-2

Arisaka, T., Mitsumata, M., Kawasumi, M., Tohjima, T., Hirose, S., and Yoshida, Y. (1995). Effects of shear stress on glycosaminoglycan synthesis in vascular endothelial cells. Ann. N. Y. Acad. Sci. 748, 543-554. doi: 10.1111/j.1749-6632. 1994.tb17359.x

Aruffo, A., Stamenkovic, I., Melnick, M., Underhill, C. B., and Seed, B. (1990). CD44 is the principal cell surface receptor for hyaluronate. Cell 61, 1303-1313. doi: 10.1016/0092-8674(90)90694-A

Baldwin, A. L., and Thurston, G. (2001). Mechanics of endothelial cell architecture and vascular permeability. Crit. Rev. Biomed. Eng. 29, 247-278. doi: 10.1615/ CritRevBiomedEng.v29.i2.20

Bartold, P. M., Wiebkin, O. W., and Thonard, J. C. (1984). The effect of oxygen-derived free radicals on gingival proteoglycans and hyaluronic acid. J. Periodontal. Res. 19, 390-400. doi: 10.1111/j.1600-0765.1984.tb01012.x

Bartosch, A. M. W., Mathews, R., and Tarbell, J. M. (2017). Endothelial glycocalyxmediated nitric oxide production in response to selective AFM pulling. Biophys. J. 113, 101-108. doi: 10.1016/j.bpj.2017.05.033

Bass, M. D., Morgan, M. R., and Humphries, M. J. (2007). Integrins and syndecan4 make distinct, but critical, contributions to adhesion contact formation. Soft Matter. 3, 372-376. doi: 10.1039/b614610d

Becker, B. F., Jacob, M., Leipert, S., Salmon, A. H., and Chappell, D. (2015). Degradation of the endothelial glycocalyx in clinical settings: searching for the sheddases. Br. J. Clin. Pharmacol. 80, 389-402. doi: 10.1111/bcp. 12629

Becker, M., Menger, M. D., and Lehr, H. A. (1994). Heparin-released superoxide dismutase inhibits postischemic leukocyte adhesion to venular endothelium. Am. J. Physiol. 267, H925-H930. doi: 10.1152/ajpheart.1994.267.3. $\mathrm{H} 925$

Bernfield, M., Kokenyesi, R., Kato, M., Hinkes, M. T., Spring, J., Gallo, R. L., et al. (1992). Biology of the syndecans: a family of transmembrane heparan sulfate proteoglycans. Annu. Rev. Cell Biol. 8, 365-393. doi: 10.1146/annurev.cb.08. 110192.002053

Bobardt, M. D., Saphire, A. C., Hung, H. C., Yu, X., Van Der Schueren, B., Zhang, Z., et al. (2003). Syndecan captures, protects, and transmits HIV to T lymphocytes. Immunity 18, 27-39. doi: 10.1016/S1074-7613(02)00504-6

Carey, D. J., Bendt, K. M., and Stahl, R. C. (1996). The cytoplasmic domain of syndecan-1 is required for cytoskeleton association but not detergent insolubility. identification of essential cytoplasmic domain residues. J. Biol. Chem. 271, 15253-15260. doi: 10.1074/jbc.271.25.15253 throughout all levels of development. All authors approved the final version for publication.

\section{FUNDING}

This work was supported by the National Institutes of Health grants R35 HL1150732 and GM097270 (to SY).

Chappell, D., Jacob, M., Rehm, M., Stoeckelhuber, M., Welsch, U., Conzen, P., et al. (2008). Heparinase selectively sheds heparan sulphate from the endothelial glycocalyx. Biol. Chem. 389, 79-82. doi: 10.1515/BC.2008.005

Chen, H. R., Chao, C. H., Liu, C. C., Ho, T. S., Tsai, H. P., Perng, G. C., et al. (2018). Macrophage migration inhibitory factor is critical for dengue NS1-induced endothelial glycocalyx degradation and hyperpermeability. PLoS Pathog. 14:e1007033. doi: 10.1371/journal.ppat.1007033

Clausen, T. M., Sandoval, D. R., Spliid, C. B., Pihl, J., Perrett, H. R., Painter, C. D., et al. (2020). SARS-CoV-2 infection depends on cellular heparan sulfate and ACE2. Cell 183, 1043-1057 e1015.

Connolly-Andersen, A. M., Thunberg, T., and Ahlm, C. (2014). Endothelial activation and repair during hantavirus infection: association with disease outcome. Open Forum Infect. Dis. 1:ofu027. doi: 10.1093/ofid/ofu027

Constantinescu, A. A., Vink, H., and Spaan, J. A. (2003). Endothelial cell glycocalyx modulates immobilization of leukocytes at the endothelial surface. Arterioscler. Thromb. Vasc. Biol. 23, 1541-1547. doi: 10.1161/01.ATV.0000085630.243 53.3D

Coste, B., Mathur, J., Schmidt, M., Earley, T. J., Ranade, S., Petrus, M. J., et al. (2010). Piezo1 and Piezo2 are essential components of distinct mechanically activated cation channels. Science 330, 55-60. doi: 10.1126/science.1193270

Curry, F. E., and Adamson, R. H. (2012). Endothelial glycocalyx: permeability barrier and mechanosensor. Ann. Biomed. Eng. 40, 828-839. doi: 10.1007/ s10439-011-0429-8

Curry, F. E., and Michel, C. C. (1980). A fiber matrix model of capillary permeability. Microvasc. Res. 20, 96-99. doi: 10.1016/0026-2862(80)90024-2

Curry, F. R. (2005). Microvascular solute and water transport. Microcirculation 12, 17-31. doi: 10.1080/10739680590894993

Davies, P. F. (1995). Flow-mediated endothelial mechanotransduction. Physiol. Rev. 75, 519-560. doi: 10.1152/physrev.1995.75.3.519

de Mesy Bentley, K. L. (2011). An 11-mum-thick glycocalyx?: it's all in the technique! Arterioscler. Thromb. Vasc. Biol. 31, 1712-1713. doi: 10.1161/ ATVBAHA.111.229849

Dimmeler, S., Fleming, I., Fisslthaler, B., Hermann, C., Busse, R., and Zeiher, A. M. (1999). Activation of nitric oxide synthase in endothelial cells by Akt-dependent phosphorylation. Nature 399, 601-605. doi: 10.1038/21224

Dragovich, M. A., Chester, D., Fu, B. M., Wu, C., Xu, Y., Goligorsky, M. S., et al. (2016). Mechanotransduction of the endothelial glycocalyx mediates nitric oxide production through activation of TRP channels. Am. J. Physiol. Cell Physiol. 311, C846-C853. doi: 10.1152/ajpcell.00288.2015

Ebong, E. E., Lopez-Quintero, S. V., Rizzo, V., Spray, D. C., and Tarbell, J. M. (2014). Shear-induced endothelial NOS activation and remodeling via heparan sulfate, glypican-1, and syndecan-1. Integr. Biol. 6, 338-347. doi: 10.1039/ C3IB40199E

Ebong, E. E., Macaluso, F. P., Spray, D. C., and Tarbell, J. M. (2011). Imaging the endothelial glycocalyx in vitro by rapid freezing/freeze substitution transmission electron microscopy. Arterioscler. Thromb. Vasc. Biol. 31, 19081915. doi: 10.1161/ATVBAHA.111.225268

Echtermeyer, F., Baciu, P. C., Saoncella, S., Ge, Y., and Goetinck, P. F. (1999). Syndecan- 4 core protein is sufficient for the assembly of focal adhesions and actin stress fibers. J. Cell Sci. 112(Pt 20), 3433-3441. doi: 10.1242/jcs.112.20. 3433

Esko, J. D., Kimata, K., and Lindahl, U. (2009). "Proteoglycans and sulfated glycosaminoglycans," in Essentials of Glycobiology, eds A. Nd, R. D. Varki, J. D. Cummings, H. H. Esko, and P. Freeze. (New York: Cold Spring Harbor).

Fan, J., Sun, Y., Xia, Y., Tarbell, J. M., and Fu, B. M. (2019). Endothelial surface glycocalyx (ESG) components and ultra-structure revealed by stochastic optical 
reconstruction microscopy (STORM). Biorheology 56, 77-88. doi: 10.3233/ BIR-180204

Florian, J. A., Kosky, J. R., Ainslie, K., Pang, Z., Dull, R. O., and Tarbell, J. M. (2003). Heparan sulfate proteoglycan is a mechanosensor on endothelial cells. Circ. Res. 93, e136-e142. doi: 10.1161/01.RES.0000101744.47866.D5

Fu, B. M., and Tarbell, J. M. (2013). Mechano-sensing and transduction by endothelial surface glycocalyx: composition, structure, and function. Wiley Interdiscip. Rev. Syst. Biol. Med. 5, 381-390. doi: 10.1002/wsbm.1211

Gallay, P. (2004). Syndecans and HIV-1 pathogenesis. Microbes Infect. 6, 617-622. doi: 10.1016/j.micinf.2004.02.004

Gao, Y., and Galis, Z. S. (2021). Exploring the role of endothelial cell resilience in cardiovascular health and disease. Arterioscler. Thromb. Vasc. Biol. 41, 179-185. doi: 10.1161/ATVBAHA.120.314346

Glasner, D. R., Ratnasiri, K., Puerta-Guardo, H., Espinosa, D. A., Beatty, P. R., and Harris, E. (2017). Dengue virus NS1 cytokine-independent vascular leak is dependent on endothelial glycocalyx components. PLoS Pathog. 13:e1006673. doi: 10.1371/journal.ppat.1006673

Gouverneur, M., Spaan, J. A., Pannekoek, H., Fontijn, R. D., and Vink, H. (2006). Fluid shear stress stimulates incorporation of hyaluronan into endothelial cell glycocalyx. Am. J. Physiol. Heart Circ. Physiol. 290, H458-H452. doi: 10.1152/ ajpheart.00592.2005

Greenwald, R. A., and Moy, W. W. (1980). Effect of oxygen-derived free radicals on hyaluronic acid. Arthritis Rheum. 23, 455-463. doi: 10.1002/art.1780230408

Halliwell, B. (1978). Superoxide-dependent formation of hydroxyl radicals in the presence of iron salts. its role in degradation of hyaluronic acid by a superoxidegenerating system. FEBS Lett. 96, 238-242. doi: 10.1016/0014-5793(78)80 409-8

Han, S., Lee, S. J., Kim, K. E., Lee, H. S., Oh, N., Park, I., et al. (2016). Amelioration of sepsis by TIE2 activation-induced vascular protection. Sci. Transl. Med. 8:335ra55. doi: 10.1126/scitranslmed.aad9260

Haywood-Watson, R. J., Holcomb, J. B., Gonzalez, E. A., Peng, Z., Pati, S., Park, P. W., et al. (2011). Modulation of syndecan-1 shedding after hemorrhagic shock and resuscitation. PLoS One 6:e23530. doi: 10.1371/journal.pone. 0023530

Henry, C. B., and Duling, B. R. (1999). Permeation of the luminal capillary glycocalyx is determined by hyaluronan. Am. J. Physiol. 277, H508-H514. doi: 10.1152/ajpheart.1999.277.2.H508

Hippensteel, J. A., Anderson, B. J., Orfila, J. E., Mcmurtry, S. A., Dietz, R. M., Su, G., et al. (2019a). Circulating heparan sulfate fragments mediate septic cognitive dysfunction. J. Clin. Invest. 129, 1779-1784. doi: 10.1172/JCI124 485

Hippensteel, J. A., Uchimido, R., Tyler, P. D., Burke, R. C., Han, X., Zhang, F., et al. (2019b). Intravenous fluid resuscitation is associated with septic endothelial glycocalyx degradation. Crit. Care 23:259. doi: 10.1186/s13054-0192534-2

Huxley, V. H., and Curry, F. E. (1985). Albumin modulation of capillary permeability: test of an adsorption mechanism. Am. J. Physiol. 248, H264-H273. doi: 10.1152/ajpheart.1985.248.2.H264

Ihrcke, N. S., Wrenshall, L. E., Lindman, B. J., and Platt, J. L. (1993). Role of heparan sulfate in immune system-blood vessel interactions. Immunol. Today 14, 500-505. doi: 10.1016/0167-5699(93)90265-M

Jacob, M., Bruegger, D., Rehm, M., Welsch, U., Conzen, P., and Becker, B. F. (2006). Contrasting effects of colloid and crystalloid resuscitation fluids on cardiac vascular permeability. Anesthesiology 104, 1223-1231. doi: 10.1097/00000542200606000-00018

Jambusaria, A., Hong, Z., Zhang, L., Srivastava, S., Jana, A., Toth, P. T., et al. (2020). Endothelial heterogeneity across distinct vascular beds during homeostasis and inflammation. Elife 9:e51413. doi: 10.7554/eLife.51413

Johansson, P. I., Sorensen, A. M., Perner, A., Welling, K. L., Wanscher, M., Larsen, C. F., et al. (2011a). Disseminated intravascular coagulation or acute coagulopathy of trauma shock early after trauma? an observational study. Crit. Care 15:R272. doi: 10.1186/cc10553

Johansson, P. I., Stensballe, J., Rasmussen, L. S., and Ostrowski, S. R. (2011b). A high admission syndecan-1 level, a marker of endothelial glycocalyx degradation, is associated with inflammation, protein $\mathrm{C}$ depletion, fibrinolysis, and increased mortality in trauma patients. Ann. Surg. 254, 194-200. doi: 10.1097/SLA.0b013e318226113d
Johnson, G. B., Brunn, G. J., Kodaira, Y., and Platt, J. L. (2002). Receptor-mediated monitoring of tissue well-being via detection of soluble heparan sulfate by Tolllike receptor 4. J. Immunol. 168, 5233-5239. doi: 10.4049/jimmunol.168.10. 5233

Jung, F., Kruger-Genge, A., Franke, R. P., Hufert, F., and Kupper, J. H. (2020). COVID-19 and the endothelium. Clin. Hemorheol. Microcirc. 75, 7-11. doi: $10.3233 / \mathrm{CH}-209007$

Kaur, S., Tripathi, D. M., and Yadav, A. (2020). The enigma of endothelium in COVID-19. Front. Physiol. 11:989. doi: 10.3389/fphys.2020.00989

Kozar, R. A., Peng, Z., Zhang, R., Holcomb, J. B., Pati, S., Park, P., et al. (2011). Plasma restoration of endothelial glycocalyx in a rodent model of hemorrhagic shock. Anesth. Analg. 112, 1289-1295. doi: 10.1213/ANE.0b013e318210385c

Kutuzov, N., Flyvbjerg, H., and Lauritzen, M. (2018). Contributions of the glycocalyx, endothelium, and extravascular compartment to the blood-brain barrier. Proc. Natl. Acad. Sci. U. S. A. 115, E9429-E9438. doi: 10.1073/pnas. 1802155115

Lennon, F. E., and Singleton, P. A. (2011). Hyaluronan regulation of vascular integrity. Am. J. Cardiovasc. Dis. 1, 200-213.

Li, L., Ly, M., and Linhardt, R. J. (2012). Proteoglycan sequence. Mol. Biosyst. 8, 1613-1625. doi: 10.1039/c2mb25021g

Li, Q., Xie, Y., Wong, M., and Lebrilla, C. B. (2019). Characterization of cell Glycocalyx with mass spectrometry methods. Cells 8:882. doi: 10.3390/ cells 8080882

Li, Q., Xie, Y., Wong, M., Barboza, M., and Lebrilla, C. B. (2020). Comprehensive structural glycomic characterization of the glycocalyxes of cells and tissues. Nat. Protoc. 15, 2668-2704. doi: 10.1038/s41596-020-0350-4

Libby, P., and Luscher, T. (2020). COVID-19 is, in the end, an endothelial disease. Eur. Heart J. 41, 3038-3044. doi: 10.1093/eurheartj/ehaa623

Lieleg, O., Baumgartel, R. M., and Bausch, A. R. (2009). Selective filtering of particles by the extracellular matrix: an electrostatic bandpass. Biophys. J. 97, 1569-1577. doi: 10.1016/j.bpj.2009.07.009

Lipowsky, H. H., and Lescanic, A. (2013). The effect of doxycycline on shedding of the glycocalyx due to reactive oxygen species. Microvasc. Res. 90, 80-85. doi: 10.1016/j.mvr.2013.07.004

Lipowsky, H. H., Kovalcheck, S., and Zweifach, B. W. (1978). The distribution of blood rheological parameters in the microvasculature of cat mesentery. Circ. Res. 43, 738-749. doi: 10.1161/01.RES.43.5.738

Lipowsky, H. H., Sah, R., and Lescanic, A. (2011). Relative roles of doxycycline and cation chelation in endothelial glycan shedding and adhesion of leukocytes. Am. J. Physiol. Heart Circ. Physiol. 300, H415-H422. doi: 10.1152/ajpheart.00923. 2010

Lipowsky, H. H., Usami, S., and Chien, S. (1980). In vivo measurements of "apparent viscosity" and microvessel hematocrit in the mesentery of the cat. Microvasc. Res. 19, 297-319. doi: 10.1016/0026-2862(80)90050-3

Lopez-Quintero, S. V., Amaya, R., Pahakis, M., and Tarbell, J. M. (2009). The endothelial glycocalyx mediates shear-induced changes in hydraulic conductivity. Am. J. Physiol. Heart Circ. Physiol. 296, H1451-H1456. doi: 10.1152/ajpheart.00894.2008

Luft, J. H. (1966). Fine structures of capillary and endocapillary layer as revealed by ruthenium red. Fed. Proc. 25, 1773-1783.

Luker, J. N., Vigiola Cruz, M., Carney, B. C., Day, A., Moffatt, L. T., Johnson, L. S., et al. (2018). Shedding of the endothelial glycocalyx is quantitatively proportional to burn injury severity. Ann. Burns Fire Disasters 31, 17-22.

Luplertlop, N., and Misse, D. (2008). MMP cellular responses to dengue virus infection-induced vascular leakage. Jpn. J. Infect. Dis. 61, 298-301.

Manon-Jensen, T., Multhaupt, H. A., and Couchman, J. R. (2013). Mapping of matrix metalloproteinase cleavage sites on syndecan-1 and syndecan-4 ectodomains. FEBS J. 280, 2320-2331. doi: 10.1111/febs.12174

Marsac, D., Garcia, S., Fournet, A., Aguirre, A., Pino, K., Ferres, M., et al. (2011). Infection of human monocyte-derived dendritic cells by ANDES Hantavirus enhances pro-inflammatory state, the secretion of active MMP-9 and indirectly enhances endothelial permeability. Virol. J. 8:223. doi: 10.1186/1743-422X-8223

Martinac, B. (2004). Mechanosensitive ion channels: molecules of mechanotransduction. J. Cell Sci. 117, 2449-2460. doi: 10.1242/jcs.01232

Matrosovich, M. N., Gambaryan, A. S., Tuzikov, A. B., Byramova, N. E., Mochalova, L. V., Golbraikh, A. A., et al. (1993). Probing of the receptor-binding sites of the 
$\mathrm{H} 1$ and $\mathrm{H} 3$ influenza $\mathrm{A}$ and influenza B virus hemagglutinins by synthetic and natural sialosides. Virology 196, 111-121. doi: 10.1006/viro.1993.1459

Mederos y Schnitzler, M., Storch, U., Meibers, S., Nurwakagari, P., Breit, A., Essin, K., et al. (2008). Gq-coupled receptors as mechanosensors mediating myogenic vasoconstriction. EMBO J. 27, 3092-3103. doi: 10.1038/emboj.2008. 233

Megens, R. T., Reitsma, S., Schiffers, P. H., Hilgers, R. H., De Mey, J. G., Slaaf, D. W., et al. (2007). Two-photon microscopy of vital murine elastic and muscular arteries. combined structural and functional imaging with subcellular resolution. J. Vasc. Res. 44, 87-98. doi: 10.1159/000098259

Mica, L., Simmen, H., Werner, C. M., Plecko, M., Keller, C., Wirth, S. H., et al. (2016). Fresh frozen plasma is permissive for systemic inflammatory response syndrome, infection, and sepsis in multiple-injured patients. Am. J. Emerg. Med. 34, 1480-1485. doi: 10.1016/j.ajem.2016.04.041

Mochizuki, S., Vink, H., Hiramatsu, O., Kajita, T., Shigeto, F., Spaan, J. A., et al. (2003). Role of hyaluronic acid glycosaminoglycans in shear-induced endothelium-derived nitric oxide release. Am. J. Physiol. Heart Circ. Physiol. 285, H722-H726. doi: 10.1152/ajpheart.00691.2002

Moseley, R., Waddington, R. J., and Embery, G. (1997). Degradation of glycosaminoglycans by reactive oxygen species derived from stimulated polymorphonuclear leukocytes. Biochim. Biophys. Acta 1362, 221-231. doi: 10.1016/S0925-4439(97)00083-5

Moseley, R., Waddington, R., Evans, P., Halliwell, B., and Embery, G. (1995). The chemical modification of glycosaminoglycan structure by oxygen-derived species in vitro. Biochim. Biophys. Acta 1244, 245-252. doi: 10.1016/03044165(95)00010-9

Multhaupt, H. A., Yoneda, A., Whiteford, J. R., Oh, E. S., Lee, W., and Couchman, J. R. (2009). Syndecan signaling: when, where and why? J. Physiol. Pharmacol. $60,31-38$.

Nelson, A., Berkestedt, I., Schmidtchen, A., Ljunggren, L., and Bodelsson, M. (2008). Increased levels of glycosaminoglycans during septic shock: relation to mortality and the antibacterial actions of plasma. Shock 30, 623-627. doi: 10.1097/SHK.0b013e3181777da3

Nikolian, V. C., Dekker, S. E., Bambakidis, T., Higgins, G. A., Dennahy, I. S., Georgoff, P. E., et al. (2018). Improvement of blood-brain barrier integrity in traumatic brain injury and hemorrhagic shock following treatment with valproic acid and fresh Frozen Plasma. Crit. Care Med. 46, e59-e66. doi: 10.1097/CCM.0000000000002800

Okada, H., Takemura, G., Suzuki, K., Oda, K., Takada, C., Hotta, Y., et al. (2017). Three-dimensional ultrastructure of capillary endothelial glycocalyx under normal and experimental endotoxemic conditions. Crit. Care 21:261. doi: 10.1186/s13054-017-1841-8

Peng, Z., Pati, S., Potter, D., Brown, R., Holcomb, J. B., Grill, R., et al. (2013). Fresh frozen plasma lessens pulmonary endothelial inflammation and hyperpermeability after hemorrhagic shock and is associated with loss of syndecan 1. Shock 40, 195-202. doi: 10.1097/SHK.0b013e31829f91fc

Pohl, U., Herlan, K., Huang, A., and Bassenge, E. (1991). EDRF-mediated shearinduced dilation opposes myogenic vasoconstriction in small rabbit arteries. Am. J. Physiol. 261, H2016-H2023. doi: 10.1152/ajpheart.1991.261.6.H2016

Potter, D. R., and Damiano, E. R. (2008). The hydrodynamically relevant endothelial cell glycocalyx observed in vivo is absent in vitro. Circ. Res. 102, 770-776. doi: 10.1161/CIRCRESAHA.107.160226

Potter, D. R., Jiang, J., and Damiano, E. R. (2009). The recovery time course of the endothelial cell glycocalyx in vivo and its implications in vitro. Circ. Res. 104, 1318-1325. doi: 10.1161/CIRCRESAHA.108.191585

Pries, A. R., and Kuebler, W. M. (2006). Normal endothelium. in: moncasa, S., higgs, A. the vascular endothelium I. Handb. Exp. Pharmacol. 176, 1-40. doi: 10.1007/3-540-32967-6_1

Puerta-Guardo, H., Glasner, D. R., and Harris, E. (2016). Dengue virus NS1 disrupts the endothelial glycocalyx, leading to hyperpermeability. PLoS Pathog. 12:e1005738. doi: 10.1371/journal.ppat.1005738

Rehm, M., Zahler, S., Lotsch, M., Welsch, U., Conzen, P., Jacob, M., et al. (2004). Endothelial glycocalyx as an additional barrier determining extravasation of $6 \%$ hydroxyethyl starch or $5 \%$ albumin solutions in the coronary vascular bed. Anesthesiology 100, 1211-1223. doi: 10.1097/00000542-200405000-00025

Reitsma, S., Slaaf, D. W., Vink, H., Van Zandvoort, M. A., and Oude Egbrink, M. G. (2007). The endothelial glycocalyx: composition, functions, and visualization. Pflugers Arch. 454, 345-359. doi: 10.1007/s00424-007-0212-8
Riley, N. M., Bertozzi, C. R., and Pitteri, S. J. (2020). A pragmatic guide to enrichment strategies for mass spectrometry-based glycoproteomics. Mol. Cell. Proteomics 20:100029. doi: 10.1074/mcp.R120.002277

Ringer, P., Colo, G., Fassler, R., and Grashoff, C. (2017). Sensing the mechanochemical properties of the extracellular matrix. Matrix Biol. 64, 6-16. doi: 10.1016/j.matbio.2017.03.004

Rizzo, V., Mcintosh, D. P., Oh, P., and Schnitzer, J. E. (1998). In situ flow activates endothelial nitric oxide synthase in luminal caveolae of endothelium with rapid caveolin dissociation and calmodulin association. J. Biol. Chem. 273, 34724-34729. doi: $10.1074 /$ jbc.273.52.34724

Roth, J. (1983). Application of lectin-gold complexes for electron microscopic localization of glycoconjugates on thin sections. J. Histochem. Cytochem. 31, 987-999. doi: 10.1177/31.8.6190857

Russell, R. J., Kerry, P. S., Stevens, D. J., Steinhauer, D. A., Martin, S. R., Gamblin, S. J., et al. (2008). Structure of influenza hemagglutinin in complex with an inhibitor of membrane fusion. Proc. Natl. Acad. Sci. U. S. A. 105, 17736-17741. doi: 10.1073/pnas.0807142105

Sallisalmi, M., Tenhunen, J., Yang, R., Oksala, N., and Pettila, V. (2012). Vascular adhesion protein-1 and syndecan-1 in septic shock. Acta Anaesthesiol. Scand. 56, 316-322. doi: 10.1111/j.1399-6576.2011.02578.x

Saphire, A. C., Bobardt, M. D., Zhang, Z., David, G., and Gallay, P. A. (2001). Syndecans serve as attachment receptors for human immunodeficiency virus type 1 on macrophages. J. Virol. 75, 9187-9200. doi: 10.1128/JVI.75.19.91879200.2001

Schmidt, E. P., Yang, Y., Janssen, W. J., Gandjeva, A., Perez, M. J., Barthel, L., et al. (2012). The pulmonary endothelial glycocalyx regulates neutrophil adhesion and lung injury during experimental sepsis. Nat. Med. 18, 1217-1223. doi: $10.1038 / \mathrm{nm} .2843$

Schnitzer, J. E. (1988). Glycocalyx electrostatic potential profile analysis: ion, $\mathrm{pH}$, steric, and charge effects. Yale J. Biol. Med. 61, 427-446.

Singh, A., Ramnath, R. D., Foster, R. R., Wylie, E. C., Friden, V., Dasgupta, I., et al. (2013). Reactive oxygen species modulate the barrier function of the human glomerular endothelial glycocalyx. PLoS One 8:e55852. doi: 10.1371/journal. pone. 0055852

Stringer, S. E., and Gallagher, J. T. (1997). Heparan sulphate. Int. J. Biochem. Cell Biol. 29, 709-714. doi: 10.1016/S1357-2725(96)00170-7

Suwarto, S., Sasmono, R. T., Sinto, R., Ibrahim, E., and Suryamin, M. (2017). Association of endothelial glycocalyx and tight and adherens junctions with severity of plasma leakage in dengue infection. J. Infect. Dis. 215, 992-999. doi: 10.1093/infdis/jix041

Suzuki, Y. (2003). [Receptor binding specificity of influenza virus and its budding from the host cells]. Tanpakushitsu Kakusan Koso 48, 1141-1146.

Tang, T. H., Alonso, S., Ng, L. F., Thein, T. L., Pang, V. J., Leo, Y. S., et al. (2017). Increased serum hyaluronic acid and heparan sulfate in dengue fever: association with plasma leakage and disease severity. Sci. Rep. 7:46191. doi: 10.1038/srep46191

Tarbell, J. M., and Ebong, E. E. (2008). The endothelial glycocalyx: a mechanosensor and -transducer. Sci. Signal. 1:t8. doi: 10.1126/scisignal.140pt8

Tarbell, J. M., and Pahakis, M. Y. (2006). Mechanotransduction and the glycocalyx. J. Intern. Med. 259, 339-350. doi: 10.1111/j.1365-2796.2006.01620.x

Teuwen, L. A., Geldhof, V., Pasut, A., and Carmeliet, P. (2020). COVID-19: the vasculature unleashed. Nat. Rev. Immunol. 20, 389-391. doi: 10.1038/s41577020-0343-0

Uchimido, R., Schmidt, E. P., and Shapiro, N. I. (2019). The glycocalyx: a novel diagnostic and therapeutic target in sepsis. Crit. Care 23:16. doi: 10.1186/ s13054-018-2292-6

van den Berg, B. M., Vink, H., and Spaan, J. A. (2003). The endothelial glycocalyx protects against myocardial edema. Circ. Res. 92, 592-594. doi: 10.1161/01.RES. 0000065917.53950 .75

van Haaren, P. M., Vanbavel, E., Vink, H., and Spaan, J. A. (2003). Localization of the permeability barrier to solutes in isolated arteries by confocal microscopy. Am. J. Physiol. Heart Circ. Physiol. 285, H2848-H2856. doi: 10.1152/ajpheart. 00117.2003

Vink, H., and Duling, B. R. (2000). Capillary endothelial surface layer selectively reduces plasma solute distribution volume. Am. J. Physiol. Heart Circ. Physiol. 278, H285-H289. doi: 10.1152/ajpheart.2000.278.1.H285

Vink, H., Constantinescu, A. A., and Spaan, J. A. (2000). Oxidized lipoproteins degrade the endothelial surface layer : implications for platelet-endothelial 
cell adhesion. Circulation 101, 1500-1502. doi: 10.1161/01.CIR.101.13. 1500

Voyvodic, P. L., Min, D., Liu, R., Williams, E., Chitalia, V., Dunn, A. K., et al. (2014). Loss of syndecan-1 induces a pro-inflammatory phenotype in endothelial cells with a dysregulated response to atheroprotective flow. J. Biol. Chem. 289, 9547-9559. doi: 10.1074/jbc.M113.541573

Wang, C., Puerta-Guardo, H., Biering, S. B., Glasner, D. R., Tran, E. B., Patana, M., et al. (2019). Endocytosis of flavivirus NS1 is required for NS1-mediated endothelial hyperpermeability and is abolished by a single $\mathrm{N}$-glycosylation site mutation. PLoS Pathog. 15:e1007938. doi: 10.1371/journal.ppat.1007938

Weinbaum, S., Tarbell, J. M., and Damiano, E. R. (2007). The structure and function of the endothelial glycocalyx layer. Annu. Rev. Biomed. Eng. 9, 121167. doi: 10.1146/annurev.bioeng.9.060906.151959

Weis, W., Brown, J. H., Cusack, S., Paulson, J. C., Skehel, J. J., and Wiley, D. C. (1988). Structure of the influenza virus haemagglutinin complexed with its receptor, sialic acid. Nature 333, 426-431. doi: 10.1038/333426a0

Xu, D., Young, J. H., Krahn, J. M., Song, D., Corbett, K. D., Chazin, W. J., et al. (2013). Stable RAGE-heparan sulfate complexes are essential for signal transduction. ACS Chem. Biol. 8, 1611-1620. doi: 10.1021/cb4001553

$\mathrm{Xu}$, D., Young, J., Song, D., and Esko, J. D. (2011). Heparan sulfate is essential for high mobility group protein 1 (HMGB1) signaling by the receptor for advanced glycation end products (RAGE). J. Biol. Chem. 286, 41736-41744. doi: 10.1074/jbc.M111.299685

Yamaoka-Tojo, M. (2020). Endothelial glycocalyx damage as a systemic inflammatory microvascular endotheliopathy in COVID-19. Biomed. J. 43, 399-413. doi: 10.1016/j.bj.2020.08.007

Yang, X., Meegan, J. E., Jannaway, M., Coleman, D. C., and Yuan, S. Y. (2018). A disintegrin and metalloproteinase 15-mediated glycocalyx shedding contributes to vascular leakage during inflammation. Cardiovasc. Res. 114, 1752-1763. doi: $10.1093 / \mathrm{cvr} / \mathrm{cvy} 167$

Yen, W., Cai, B., Yang, J., Zhang, L., Zeng, M., Tarbell, J. M., et al. (2015). Endothelial surface glycocalyx can regulate flow-induced nitric oxide production in microvessels in vivo. PLoS One 10:e0117133. doi: 10.1371/ journal.pone.0117133

Yoon, J. H., Lee, E. S., and Jeong, Y. (2017). In vivo imaging of the cerebral endothelial Glycocalyx in mice. J. Vasc. Res. 54, 59-67. doi: 10.1159/000457799

Zehtabchi, S., and Nishijima, D. K. (2009). Impact of transfusion of fresh-frozen plasma and packed red blood cells in a 1:1 ratio on survival of emergency department patients with severe trauma. Acad. Emerg. Med. 16, 371-378. doi: $10.1111 / \mathrm{j} .1553-2712.2009 .00386 . x$

Zeng, Y., and Liu, J. (2016). Role of glypican-1 in endothelial NOS activation under various steady shear stress magnitudes. Exp. Cell Res. 348, 184-189. doi: 10.1016/j.yexcr.2016.09.017

Zeng, Y., and Tarbell, J. M. (2014). The adaptive remodeling of endothelial glycocalyx in response to fluid shear stress. PLoS One 9:e86249. doi: 10.1371/ journal.pone.0086249

Zeng, Y., Zhang, X. F., Fu, B. M., and Tarbell, J. M. (2018). The role of endothelial surface Glycocalyx in mechanosensing and transduction. Adv. Exp. Med. Biol. 1097, 1-27. doi: 10.1007/978-3-319-964 45-4_1

Zou, Y., Akazawa, H., Qin, Y., Sano, M., Takano, H., Minamino, T., et al. (2004). Mechanical stress activates angiotensin II type 1 receptor without the involvement of angiotensin II. Nat. Cell Biol. 6, 499-506. doi: 10.1038/ncb1137

Conflict of Interest: The authors declare that the research was conducted in the absence of any commercial or financial relationships that could be construed as a potential conflict of interest.

Copyright (c) 2021 Villalba, Baby and Yuan. This is an open-access article distributed under the terms of the Creative Commons Attribution License (CC BY). The use, distribution or reproduction in other forums is permitted, provided the original author(s) and the copyright owner(s) are credited and that the original publication in this journal is cited, in accordance with accepted academic practice. No use, distribution or reproduction is permitted which does not comply with these terms. 